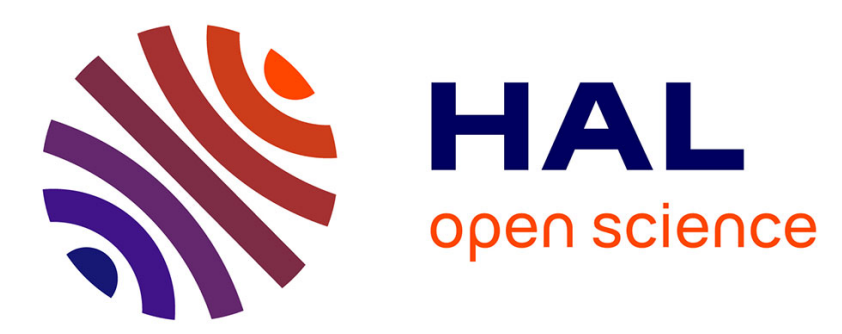

\title{
Seeing the Unseen: Revealing Mobile Malware Hidden Communications via Energy Consumption and Artificial Intelligence
}

Luca Caviglione, Mauro Gaggero, Jean-François Lalande, Wojciech Mazurczyk, Marcin Urbanski

\section{To cite this version:}

Luca Caviglione, Mauro Gaggero, Jean-François Lalande, Wojciech Mazurczyk, Marcin Urbanski. Seeing the Unseen: Revealing Mobile Malware Hidden Communications via Energy Consumption and Artificial Intelligence. IEEE Transactions on Information Forensics and Security, 2016, 11 (4), pp.799-810. 10.1109/TIFS.2015.2510825 . hal-01247495

\author{
HAL Id: hal-01247495 \\ https://hal.science/hal-01247495
}

Submitted on 22 Dec 2015

HAL is a multi-disciplinary open access archive for the deposit and dissemination of scientific research documents, whether they are published or not. The documents may come from teaching and research institutions in France or abroad, or from public or private research centers.
L'archive ouverte pluridisciplinaire HAL, est destinée au dépôt et à la diffusion de documents scientifiques de niveau recherche, publiés ou non, émanant des établissements d'enseignement et de recherche français ou étrangers, des laboratoires publics ou privés. 


\title{
Seeing the Unseen: Revealing Mobile Malware Hidden Communications via Energy Consumption and Artificial Intelligence
}

\author{
Luca Caviglione, Mauro Gaggero, Jean-François Lalande, Wojciech Mazurczyk, and Marcin Urbański
}

\begin{abstract}
Modern malware uses advanced techniques to hide from static and dynamic analysis tools. To achieve stealthiness when attacking a mobile device, an effective approach is the use of a covert channel built by two colluding applications to locally exchange data. Since this process is tightly coupled with the used hiding method, its detection is a challenging task, also worsened by the very low transmission rates. As a consequence, it is important to investigate how to reveal the presence of malicious software by using general indicators such as the energy consumed by the device. In this perspective, the paper aims to spot malware covertly exchanging data by using two detection methods based on artificial intelligence tools such as neural networks and decision trees. To verify their effectiveness, seven covert channels have been implemented and tested over a measurement framework using Android devices. Experimental results show the feasibility and effectiveness of the proposed approach to detect the hidden data exchange between colluding applications.
\end{abstract}

Index Terms-Energy-based malware detection, covert channels, colluding applications, neural networks, decision trees.

\section{INTRODUCTION}

Modern malware uses advanced techniques to defeat static analysis tools or live detection systems. Even if designing a malware is nowadays considered quite common [1], the most advanced programmers try to hide malicious behaviors by using different techniques, such as the repackaging of legitimate applications or the obfuscation/ciphering of code. Besides, by automating such mechanisms, a single attacker can add malicious code to several applications that may be sent

This research was partially supported by the Polish National Science Center under grant no. 2015/18/E/ST7/00227.

L. Caviglione and M. Gaggero are with the Institute of Intelligent Systems for Automation, National Research Council of Italy, Genoa, Italy (e-mail: luca.caviglione@ge.issia.cnr.it; mauro.gaggero@cnr.it)

J.-F. Lalande is with the INSA Centre Val de Loire, Bourges, France and with the CIDRE team, CentraleSupélec/Inria, Rennes, France (e-mail: jean-francois.lalande@insa-cvl.fr).

W. Mazurczyk and M. Urbański are with the Warsaw University of Technology, Institute of Telecommunications, Warsaw, Poland (e-mail: wmazurczyk@tele.pw.edu.pl; m.urbanski@stud.elka.pw.edu.pl).

The source code of the covert channels and the measurement framework described in this paper is available online at http://steganocc.gforge.inria.fr.

(c) 2015 IEEE. Personal use of this material is permitted. Permission from IEEE must be obtained for all other uses, in any current or future media, including reprinting/republishing this material for advertising or promotional purposes, creating new collective works, for resale or redistribution to servers or lists, or reuse of any copyrighted component of this work in other works.

This article is a post-print version of the paper published in IEEE Transactions on Information Forensics and Security with DOI 10.1109/TIFS.2015.2510825 to alternative markets. As a consequence, classical signaturebased methods have limited results [2].

One of the most advanced mechanisms used by malware to exfiltrate information or to bypass the security frameworks of mobile devices relies upon information-hiding techniques to exchange data between different processes. Especially, as in the case of smartphones, a local covert channel can be used to setup a communication path between two colluding applications to extract personal information [3], [4]. As it has been observed in [5], mobile devices are particularly prone to hidden-communication attacks due to their variety of hardware resources, as they incorporate cameras, GPS, WLAN, Bluetooth, cellular networks, and many sensors. Moreover, malware developers turned a significant portion of their attention to mobile devices, leading to an increase of $1800 \%$ in mobile malware over the past two years [6]. Therefore there is an urge for research efforts to design original countermeasures and enable early prevention. Unfortunately, this is very difficult since the detection strictly depends on the type of covert channel. For instance, exploiting electromagnetic signals to covertly transmit data is very different from manipulating the statistics of the available RAM to embed secrets [5]. Additionally, covert channels typically achieve limited bandwidths, thus increasing the complexity of finding out whether a hidden exchange is ongoing.

In this perspective, a promising approach aims at exploiting general information to detect covert channels. A recent debate has emerged about the possibility of using the power consumption as an indicator to identify malicious activities. Despite [7] claims that malware cannot be detected by highlevel applications measuring energy consumption of processes, other works demonstrate that proper power measurements can reveal some threats [8]-[10].

In this paper, we show the feasibility of using measurements of the energy consumed by a device to detect malware exploiting a covert channel. To this aim, we have implemented five popular covert channels available in the literature targeting the Android platform [4], [11], together with two new ones. Further, we have developed an experimental setup to quantify the energy consumption of the software components running on a mobile device. In more details, we have used measurements provided by the high-level model of PowerTutor [8] together with values available in the / sys portion of the file system [10], [12].

To perform the detection, we developed an approach based on two well-known artificial intelligence tools, i.e., neural 
networks [13] and decision trees [14]. They are able to learn from a set of past collected energy measurements whether hidden communication is present, and then reveal threats. Specifically, two detection methods have been developed, each one using both neural networks and decision trees. The first approach requires the solution of a regression problem to predict the future behavior of energy consumption. A hidden communication is spotted if the difference between the actual and predicted consumption exceeds a certain threshold. The second method is based on a classification problem, and provides information on hidden communications by using a set of features characterizing the energy behavior of the device. The considered artificial intelligence tools have been already used to reveal malicious code [15], [16] or to prevent the execution of hazardous software on Android devices [17]. However, no previous works using energy consumption to spot information-hiding-capable malware exist in the literature.

To summarize, the main contributions of this paper are: (i) showcase the feasibility of using energy footprints for the detection of malware implementing information-hiding techniques, (ii) the creation of an ad-hoc testbed and a hybrid measurement method to characterize seven covert channels, including two new implementations, and (iii) the development of two "intelligent" frameworks to perform the detection with low computational requirements.

The rest of this paper is structured as follows. Section II reviews the literature dealing with the detection of malware by using energy measurements. Section III details the reference scenario and the considered covert channels, while Section IV describes the methodology used for the measurements of power consumption. Section V introduces the two detection methods based on neural networks and decision trees, and Section VI discusses experimental results. Finally, Section VII concludes the paper.

\section{RELATED WORKS}

Anomaly detection using energy footprints has been partially investigated in the literature, primarily for malware and network attacks. However, at best of the authors' knowledge, it has never been applied to covert channels.

In general, energy-based anomaly detection methods are grouped according to how measurements are performed. In more details, we have the following approaches.

1) System-based [7], [18]-[20]: an energy footprint is created by considering the whole consumption of the device or some specific sets of applications and/or hardware parts. The obtained data represents the "clean system" that serves as a baseline for malware discovery.

2) Application-based [21]: similarly to the previous case, an energy footprint is created for a well-defined pool of applications (e.g., games), and each one is measured separately. The collected traces are then compared at runtime against the data obtained with a single-process granularity.

3) User-based [22]-[24]: an energy footprint is created by analyzing the typical behavior of users and the related power consumption. This also includes, for instance, the applications and device features that are active, as well as their timing statistics.

4) Attack-based [9], [10], [25]-[27]: measurements are done while real attacks or malicious malware activities are performed in a controlled environment. The acquired traces form a database of energy signatures used for the detection.

It is worth noting that methods belonging to the first three groups can potentially deal with unknown threats, while the last one only allows to recognize attacks for which signatures are available.

Concerning system-based methods, Jacoby et al. [19] demonstrated how to reveal network attacks by using a battery-based Intrusion Detection System (IDS) analyzing the power consumption and the utilization levels of some critical hardware/software components like the CPU. Later, Nash et al. [20] proposed to estimate the energy footprint of a desktop computer by using a multiple linear regression model considering the CPU load, read/write accesses to the storage unit, and network transmissions. To evaluate the presence of malicious activities, the measured parameters are combined with performance data counters available in the operating system, and the results are compared with different thresholds. Such approach was proven to be effective, but its main drawback is the complexity to compute proper numerical values for the needed thresholds. Liu et al. [18] proposed the VirusMeter tool for Symbian smartphones that computes the energy profile for the whole "clean" system. The user is alerted by using a heuristic to compare the actual energy consumption with a reference value. Lastly, Hoffman et al. [7] performed comprehensive experiments with both artificial and real-world malware using observation windows of different lengths. They created energy footprints in a controlled setting for the IEEE 802.11 and 3G hardware of a smartphone, and the resulting power consumption was treated as a baseline for detection. Even if such an approach is effective, the main contribution of the work is about the noise level of tools used for measurements: the additional power consumed by a malware is often too small to be detectable with the resolution of many measurement software.

Among the techniques using application-based methods, the most notable work has been proposed by Kim et al. [21], where the power consumption is monitored to detect malware in Windows Mobile devices. Their proof-of-concept solution is based on the energy footprints of the applications running on the smartphone, which are compared against "clean" consumption templates.

Concerning methods explicitly considering the behavior of the user, Dixon et al. [22]-[24] showed that there is a strong correlation between the battery drain of a mobile device and the user's location. This can be exploited to determine the average power consumption for different locations and make the detection of abnormalities more efficient. In fact, instead of considering the required power only as a function of time, location-specific energy profiles are used as additional indicators.

Regarding attack-based approaches, Buennemeyer et al. [26] investigated the energy signatures of some network threats 
against mobile devices. In more details, the power consumption of a device is correlated with IEEE 802.11 activities. If an irregularity is discovered, it is compared with existing signatures to perform the detection of the attack. Then, each mobile device exchanges alerts with peers, thus implementing a distributed network IDS. This work has been further extended by modifying the rates at which the battery status is polled, and by considering the activity of the Bluetooth air interface to increase the performance in terms of correct detections [27]. Moreover, Caviglione and Merlo [9] focused on how antivirus and network attacks such as port scan and ping floods impact over the battery depletion of different smartphones. They state the need for "green security" mechanisms to effectively develop consumption-based malware detection systems [28]. Curti et al. [25] studied the energy footprints for benign applications like Skype or YouTube and also for network attacks like Denial of Service. They also provided a power consumption model for the hardware involved in IEEE 802.11 communications allowing to distinguish a normal traffic pattern from a network threat. This work has been further extended by Merlo et al. [10] by analyzing the feasibility of porting the two aforementioned approaches on Android devices with the aim of developing a malware detection framework. Unfortunately, the proposed solutions turned out to be unsuitable, mainly due to implementation issues, which can be overcome by introducing the direct observation of power consumption from the battery hardware without the need to dwell deeply into the drivers.

Literature also indicates that future research directions should consider hybrid approaches, i.e., the power consumption should be enriched with additional information such as the memory usage. Even if hybrid approaches are possible, they are typically implemented in a standalone fashion [19] or as a part of a larger network-based IDS (see, e.g., [26]). Furthermore, the technology evolves very dynamically, thus studies performed even few years ago could quickly become obsolete as functionality and capabilities of modern devices significantly outrun the old ones.

\section{Covert Channels}

In this section, we describe how a prototypical malware exploiting a local covert channel to secretly leak sensitive data has been developed, and how we have studied its main characteristics.

\section{A. Reference Scenario}

We consider the typical scenario depicted in Figure 1, where a malware composed of two colluding applications exchanges data through a local covert channel built within the device in order to exfiltrate sensitive information [3], [4], [11]. In more details, the process CCSender has access to the data but has not the permission to use the network. Instead, the colluding application CCReceiver has access to the network, hence it is able to exfiltrate the received data to an external server or a Command \& Control (C\&C) facility. Obviously, the communications of the CCReceiver towards the $C \& C$ could be detected, for instance, by inspecting the traffic produced

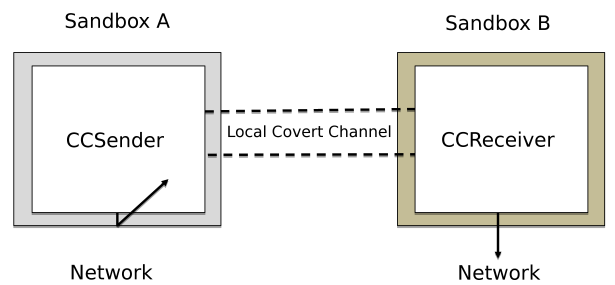

Fig. 1. Typical communication scenario of two colluding parts of a malware: CCSender and CCReceiver exchange data through a local covert channel.

by the device. Thus, it is common to use other informationhiding methods to build a network covert channel within the produced flow. For instance, some malware uses a TOR client to reach the server anonymously, making classic traffic analysis ineffective [29]. Consequently, analyzing an encrypted flow of information produced by the CCReceiver does not help to reveal the presence of a malware, and this is why this paper focuses on the detection of the local covert channel itself.

Moreover, we assume that the malware monitors the operations performed by the user in order to transmit data when he/she is not active [30]. Indeed, waking up the covert channel during user's activity could degrade the performances of the device and reveal the presence of the threat. To this aim, many modern malware delay their activation in order to be invisible or not rise attention. Thus, even if the triggering of the malware may occur at any time, it is more likely to happen when the user is not active. For example, the families of malware DroidKungFu 1 and 2 include a time bomb mechanism that triggers the malicious behavior after a predefined period of time [31]. With such a protection, a malware would be running statistically when the smartphone is idle. Therefore, we focus on such low attention-raising hazards operating when the device is idle since they are the preferred choice to perform attacks and to avoid detection [3].

\section{B. Implemented Covert Channels}

For experimental purposes we implemented on the Android platform seven local covert channels between the processes CCSender and CCReceiver. Five of them have been already proposed in the literature and they are listed as follows.

1) Type of Intent [11]: the secret receiver registers 256 types of intent listeners and the secret sender encodes data by choosing and sending an intent of the right type.

2) File Lock [32]: the secret sender communicates by locking a file. The secret receiver also tries to lock the same file and, if it succeeds, a 0 is inferred. Otherwise, an exception is raised, meaning that the secret sender has locked the file before the secret receiver. In this case, a 1 is received.

3) System Load [11]: the secret sender sends a 1 by burdening the CPU of the device. The secret receiver checks how many clock ticks the sender has got since the previous iteration. If the value is greater than a certain threshold, a 1 is inferred, a 0 otherwise.

4) Volume Settings [32]: the secret data is encoded into the ringtone volume level of the device. If the sender can use 
TABLE I

MEASURED BANDWIDTHS (IN BITS PER SECOND) FOR THE CONSIDERED COVERT CHANNELS COMPARED TO THE THEORETICAL VALUES.

\begin{tabular}{ccc}
\hline Covert Channel & Th. Bandwidth & Meas. Bandwidth \\
\hline Type of Intent & $15 \mathrm{~K}$ & 74.62 \\
File Size & $2 \mathrm{~K}$ & 73.96 \\
Memory Load & 500 & 74.00 \\
File Lock & 250 & 9.31 \\
System Load & 5 & 6.43 \\
Volume Settings & 450 & 33.39 \\
Unix Socket Discovery & 100 & 74.57 \\
\hline
\end{tabular}

eight levels of volume, it can send up to three bits per iteration.

5) Unix Socket Discovery [11]: the secret data is sent by encoding the information within the state of a socket. Specifically, a closed socket is equal to 1 , while an opened one is equal to 0 .

Additionally, we propose the following two new covert channels that up to now were only tested in a theoretical fashion.

6) File Size: the secret sender sets the size of a shared file and the secret receiver interprets it as a byte.

7) Memory Load: the secret receiver acquires the initial memory load of the secret sender. Then, the secret sender inflates the allocated data to send a 1 or releases memory to send a 0 .

Before investigating the related energy footprints, we conducted a performance evaluation to assess the correctness of the implementation, also in the perspective of removing possible power-hungry bugs that can void measurements. The literature provides theoretical limits and/or performance evaluations in a very mixed set of testbeds and with early implementations of the Android operating system and outdated smartphones. Thus, we investigated the data throughput to understand whether the available reference values are still valid. For this round of tests, we used a Samsung Galaxy SIII smartphone. Table I reports the mean bitrate achieved by each covert channel averaged over 100 repeated trials together with the estimated capacities as provided in the related references. In more details, the measured values highly differ from those provided in the literature. This is mainly due to changes in the Android platform and in the device drivers, hence confirming the high variability of performances as the result of a tight coupling between covert channels and the hardware/software architecture. Besides, the best obtained throughput is equal to $74.62 \mathrm{bit} / \mathrm{s}$ for the case of the Type of Intent covert channel, while the lowest is $6.43 \mathrm{bit} / \mathrm{s}$ for the System Load method. At least four methods have similar maximum bandwidths, which suggests that we have reached some limits within the Android platform.

\section{Measurement Methodology}

In this section we present the methodology used to measure the power consumption of the malware based on colluding applications exchanging data through covert channels. To have reliable data, we first investigated the literature to find the

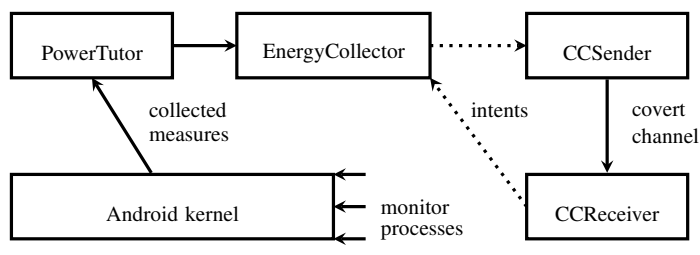

Fig. 2. Box diagram of the energy measurement architecture.

most suitable ways to gather information on the power used by applications running on Android devices. As pointed out in [10], the energy can be measured at high level using the Android APIs or at low level by probing the battery driver. Performing measures at low level is a difficult task, as it requires to patch the battery driver to get access to fine-grained data. Unfortunately, using high-level APIs may lead to values with a poor degree of reliability. For example, [10] and [12] report that high-level data is not accurate enough to detect some form of network attacks, and also proposes an alternative mechanism defined as "middle-level" exploiting information stored in the / sys portion of the file system such as the instantaneous voltage of the battery.

Based on these results, we decided not to collect lowlevel energy values for two main reasons: this technique requires deep changes in the operating system, thus lacking of portability, and has a non-negligible consumption that can mask the one of the covert channel used by the colluding applications. Instead, to quantify the power depletion we relied upon: (i) high-level energy consumption measures of each process running on the system, for which we used a modified version of PowerTutor [8], i.e., we developed a patch to enable the tool to send different process consumption to a proper data collector via an Android intent; (ii) middle-level energy consumption information acquired by gathering current and voltage values stored in / sys. We point out that since our objective is the detection of a threat without recurring to data related to the power consumed by the network subsystem, our effort of using a mixed high- and middle-level methodology is novel [33].

We developed a measurement framework both for collecting data and automatize the experiments. Figure 2 depicts the box diagram and the major interactions among the different subsystems. In particular, the EnergyCollector is the controller of the experiments and is in charge of running repeated trials and collecting data measured by PowerTutor from APIs and / sys. The latter receives gathered data each second through an intent. Obviously, the communication flow between the EnergyCollector and the CCsender/CCreceiver would not exist on a real system, and is only used to repeat the experiments. Nevertheless, after measuring the overhead, we can assume the impact of intents in terms of additional power as negligible.

For each covert channel, we conducted repeated trials according to the following flow:

1) the EnergyCollector waits a random time;

2) the EnergyCollector sends an intent to the CCsender to start a covert transmission;

3) CCsender and CCreceiver exchange a message; 
4) CCreceiver sends an intent to notify the EnergyCollector that the transmission is finished;

5) the experiment is repeated.

The random time inserted in the step 1) ensures that the beginning of the covert channel transmission is not known $a$ priori and that two different exchanges are not triggered within a given timeframe. This enables to have an unpredictable behavior, which is more coherent with real-world use cases (a discussion on malware randomly activating is provided in [34] and [35]). The duration of the transmission depends on the type of the covert channel since each one has a different bandwidth, as discussed in Section III.

\section{Energy Consumption and Black-Box Modeling}

The basic idea of our approach is to detect covert communications among colluding applications by using the energy requirements of the processes running on a device as a marker. The main benefit is the decoupling of the detection of the covert channel from its implementation. In fact, hidden communications are tightly coupled with the adopted carrier and are characterized by a very low bitrate, thus making their spotting very difficult and poorly generalizable.

To detect covert channels, two general problems have to be addressed: (i) developing an approximate model for the power consumption of a process, and (ii) using the obtained information to recognize whether a covert channel is present. Achieving such goals is challenging, especially due to the different sources of power drains, e.g., transmissions over air interfaces, memory operations, CPU- or I/O-bound behaviors, and user to kernel space switches [36]. Thus, we propose two general methods based on the black-box modeling approach.

The first technique, denoted as regression-based detection (RBD), uses a regression fed with past values of the consumption in a "clean" system to predict its future behavior. Then, if the expected energy footprint deviates too much from the real one, hidden communication is assumed present. The second approach, denoted as classification-based detection (CBD), exploits a reduced set of features describing the energetic behavior of the device. More specifically, a classification problem is solved to detect covert communications between colluding applications.

To this aim, well-known artificial intelligence tools, such as one-hidden-layer feedforward neural networks and binary decision trees, are used to model the power consumption. The RBD and CBD approaches are composed by two steps, as shown in Figure 3. First, a model of the power consumption is constructed based on a set of past collected measurements. This requires the solution of an optimization problem to find the best values of the parameters the model depends upon. Such a step is called "training", and the collected measures constitute the training set. This may be computationally demanding, but it is usually performed offline and not on the mobile device. Second, the optimized model is used to detect covert communications based on the new, actual measurements of power consumption. This step can be performed online, i.e., at runtime on the device.

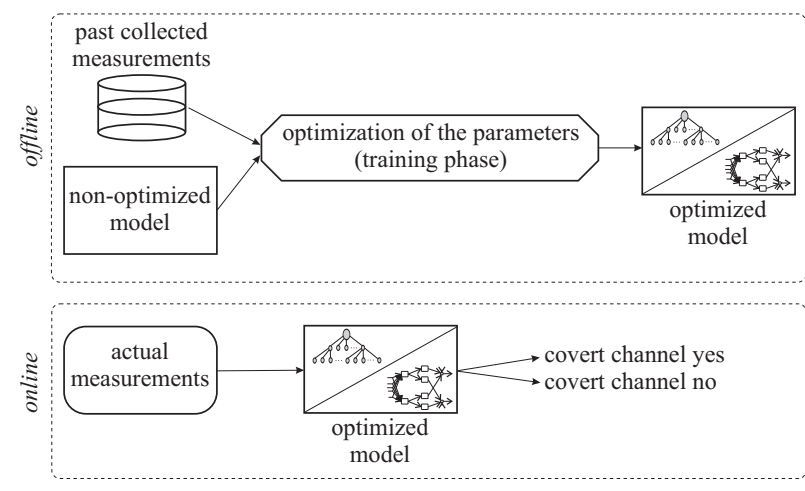

Fig. 3. Two-step procedure for the detection of covert channels.

\section{A. Neural Networks and Decision Trees}

In this section, we introduce the tools used to construct the approximate models of power consumption. Both are employed in the literature to approximate unknown relationships between input and output variables without any a-priori knowledge on the underlying dynamics. They can be used to solve either regression or classification problems [37]. Regression models map the input space into a real-valued domain. Instead, classifiers map the input space into predefined classes. The relationships between inputs and outputs are learned from the collected data and then used to associate an output to new, unseen inputs. Neural networks belong to the family of "parametric" approximators, as their output significantly depends on the parameters that define the structure of the model, whereas decision trees are usually referred to as "nonparametric" approximators, i.e., they strongly depend from the available data and, less significantly, from a reduced set of parameters.

As said, the goal is to approximate an input/output mapping $\underline{x}_{i} \mapsto \underline{y}_{i}$, where $\underline{x}_{i} \in \mathbb{R}^{n}$ is the input and $\underline{y}_{i} \in \mathbb{R}^{m}$ is the corresponding output. The unknown functional relationship between inputs and outputs is approximated by a function $\gamma$ belonging to a family $\Gamma$ of parametrized functions, i.e.,

$$
\underline{\tilde{y}_{i}}=\gamma\left(\underline{x}_{i}, \underline{\alpha}\right)
$$

where $\underline{\alpha} \in \mathbb{R}^{p}$ is a vector of parameters and $\underline{y_{i}} \in \mathbb{R}^{m}$ is the estimated value of $\underline{y}_{i}$.

In recent years, the literature of learning from data has mainly concentrated on the use of nonlinear models for the approximation of complex systems. In fact, it has been proved from both theoretical and numerical viewpoints, that classical linear models may be computationally intractable for the approximation of complex functions, especially in the case of high dimensionality of the input variables (see, e.g., [38][40] and the references therein). On the contrary, it has been shown that nonlinear structures have a greater flexibility and guarantee good approximations with a smaller number of parameters.

One-hidden-layer feedforward neural networks are a very popular nonlinear architecture already used to model a variety of systems (see, e.g., [41], [42] and references therein). In this paper we focus on neural networks with sigmoidal activation functions. They enjoy the universal approximation property, 
i.e. the ability to approximate any well-behaved function with arbitrary precision [43], [44]. The function $\gamma$ is given by a linear combination of parametrized basis functions, with a certain number of free parameters to be optimized inside. For scalar outputs, we have

$$
\gamma(\underline{x}, \underline{\alpha})=\sum_{k=1}^{\nu} c_{k} \sigma\left(\sum_{j=1}^{q} a_{k j} x_{j}+b_{k}\right)+c_{0}
$$

where $\sigma(\cdot)$ is the activation function, $\nu$ is the number of basis functions (i.e., the neurons) and $\underline{\alpha} \triangleq \operatorname{col}\left(a_{k}, b_{k}, c_{k}, c_{0}\right) \in \mathbb{R}^{p}$ is the vector of free parameters.

Concerning binary decision trees, they are widely-diffused models for learning (see, e.g., [14], [37], [45], [46] and the references therein). The output of the model is computed via a binary partition of the input space into smaller subsets called "leaves", with sides parallel to the coordinate axes, in which the values of the output are constant. Each split pursues certain performance goals and is repeated until a stopping criterion is met. The partition is called a "tree" since the leaves are added recursively with binary splits to form a tree structure. Two different types of trees exist depending on whether they are used to solve regression or classifications problems. In the case of regression trees, a constant output value is assigned to each leaf, corresponding to the average of the observed output values therein. By contrast, a certain label is assigned to the terminal leaves in classification trees. In both cases, to avoid overfitting, the obtained tree is usually "pruned" according to some regularization costs [37]. In this paper, we focus on binary trees, in which each step of a prediction involves checking the value of only one variable at a time. The functional relation $\gamma$ in (1) is then approximated by means of a piecewise-constant function over the various partitions.

After choosing the family of functions $\Gamma$, we need to find an element $\gamma^{*} \in \Gamma$ capable of reproducing at best the system behavior. This corresponds to a training procedure to optimize the parameters on the basis of the available data. To this purpose, a suitable index is used to measure the discrepancy between the estimated model output $\underline{\tilde{y}_{i}}$ for a certain value of the parameters and the real measured output values $\underline{y}_{i}$. The most used metric is a mean squared error (MSE) criterion: the optimal parameter vector $\underline{\alpha}^{*}$ is the one that minimizes the quadratic difference between the real output variables and the estimated ones, i.e.,

$$
\underline{\alpha}^{*} \triangleq \min _{\underline{\alpha} \in \mathbb{R}^{p}}\left\{\frac{1}{N} \sum_{i=1}^{N}\left(\underline{y}_{i}-\gamma\left(\underline{x}_{i}, \underline{\alpha}\right)\right)^{2}\right\}
$$

where $N$ is the number of samples used for the training.

In general, the training phase may be computationally demanding, especially for large values of $N$. However, many adhoc-developed procedures are available in the literature (see, e.g., [47], [48]). Usually, they are implemented in efficient software packages, allowing to solve the training problem in a reduced amount of time.

\section{B. Regression-Based Detection}

The RBD method is composed of two steps: the first consists in modeling the power consumed in a "clean" system, i.e., when no colluding applications performing hidden data exchanges are present in the device. To this end, a collection of past values of the consumption are used to predict the future ones. The second step is based on a comparison between the forecast consumption and the actual one. Hidden communication is spotted if the expected energy footprint deviates too much from the real one.

In more details, we define the following quantities at each sampling time $t=0,1, \ldots$. Let $p_{t} \in \mathbb{R}_{+}$be the power consumed by a process at time $t$. Such a quantity is measured and can be considered as an input. Let $w_{t+1} \in \mathbb{R}_{+}$be the prediction of the power needed by the process itself at the next time instant $t+1$, i.e., it is an output.

At least in principle, the consumption of a process at time $t+1$ may depend on the energy requirements at the previous time instants, from 0 to $t$. However, to avoid dealing with vectors of increasing dimension as the time grows, we consider a "fading memory" assumption, which consists in assuming that the output of the system (i.e., the power consumption of a process at time $t+1$ ) depends only on a finite number $q$ of past inputs [49]. In this perspective, we define a regression vector (also called regressor) as the collection of the past input variables from time $t-q+1$ up to time $t$, i.e., $\underline{p}_{t} \triangleq \operatorname{col}\left(p_{t-q+1}, p_{t-q+2}, \ldots, p_{t}\right) \in \mathbb{R}^{q}$, where $q$ is a positive constant value.

Thus, the training set for the creation of the model has the form of a set of input/output pairs

$$
\Sigma_{N} \triangleq\left\{\underline{p}_{t}, w_{t+1}\right\}_{t=1}^{N}
$$

where $N$ is the total number of available measures. The goal is to find a model that is able to capture, at each time $t$, the functional relationship between past and future consumption, i.e., the mapping $\underline{p}_{t} \mapsto w_{t+1}$. Thus, equation (1) becomes

$$
\tilde{w}_{t+1}=\gamma\left(\underline{p}_{t}, \underline{\alpha}\right)
$$

where $\tilde{w}_{t+1}$ is the estimated output at time $t+1$ and $\gamma$ is a function belonging to a family $\Gamma$ of one-hidden-layer feedforward neural networks or binary decision trees. Notice that, when the regressor is made up by long series of past observations, the dimensionality of the problem rapidly increases, hence the need of efficient approximators arises.

Once the best model within the class $\Gamma$ has been found by the offline training procedure, the second step of the RBD method requires the definition of a detection rule. Specifically, the detection is performed online by feeding at each time step $t$ the trained model with the past $q$ measurements of the consumption collected into the regressor $\underline{p}_{t-1}$. Then, the estimated consumption $\tilde{w}_{t}$ is compared with the real measured value $w_{t}$ in a time window moving over time. The same procedure is repeated at the next time steps, and a prediction error $e_{t}$ is defined as follows:

$$
e_{t} \triangleq \frac{1}{\tau} \sum_{k=t-\tau+1}^{t}\left|\tilde{w}_{k}-w_{k}\right|
$$

where $\tau$ is a given time horizon. We assume that a covert communication among two colluding applications is present if the prediction error is greater than a certain positive threshold 
$\xi$. Otherwise, it is considered absent. The rationale is that the approximate model, if properly trained, is able to predict the future behavior of the energy consumption of the "clean" system with a good level of accuracy. Hence, severe deviations reveals the presence of two processes covertly exchanging data.

Clearly, the performance of the RBD depends on the quality of the approximating model and on the parameters $q, \tau$, and $\xi$, which have to be properly tuned. We will observe their impact on the quality of detection in a real scenario in Section VI. Notice that the same model $\gamma$ can be used to detect covert communications with all the seven covert channel types described in Section III since it has been obtained using measurements obtained in a "clean" system.

\section{Classification-Based Detection}

The CBD method consists in solving a classification problem starting from a set of measurements both in the presence and in the absence of colluding applications. It requires the definition of a set of "features" representing the power consumption of the device in a concise, effective manner.

More specifically, we focus on three different features characterizing the energetic behavior of a process at each time $t$, collected into the vector $\underline{x}_{t} \in \mathbb{R}^{3}$ : (i) the average power consumption from time $t-\lambda+1$ to time $t$, where $\lambda$ is a positive constant defining a window of past measurements, (ii) the total variation of the power consumption from time $t-\lambda+1$ to time $t$, and (iii) the instantaneous consumption at time $t$. Thus, we focus on the following vector of features at each time $t$ :

$$
\underline{f}_{t} \triangleq \operatorname{col}\left(\sum_{l=t-\lambda+1}^{t} p_{l}, \sum_{l=t-\lambda+1}^{t}\left|p_{l}\right|, \quad p_{t}\right) \in \mathbb{R}^{3}
$$

Each vector $\underline{f}_{t}$ refers to a single measurement and is associated to a certain class $k$ among two possible ones, corresponding to the cases in which covert channels are used to exchanging data between colluding applications $(k=1)$ and no covert channels are established $(k=0)$.

The training set for the creation of the model takes on the form of a set of $N$ input/output pairs

$$
\Sigma_{N} \triangleq\left\{\underline{f}_{t}, g_{t}\right\}_{t=1}^{N}
$$

where the scalar output $g_{t}$ is equal to $k$ if the input vector $\underline{f}_{t}$ belongs to the class $k$.

The goal is to find a model able to recognize the class containing a given input vector that is not among the $N$ used for the training. As in the case of the RBD method, we rely upon models belonging to a certain family $\Gamma$ of one-hiddenlayer feedforward neural networks and binary decision trees, i.e., equation (1) becomes:

$$
\tilde{g}_{j}=\gamma\left(\underline{f}_{j}, \underline{\alpha}\right)
$$

where $\tilde{g}_{j}$ is the class assigned to the input vector $\underline{f}_{j}$ by the model. The output $\tilde{g}_{j}$ must be one of the two possible classes.

Clearly, the goal of the classification is to ensure that the assignment of the model is correct, i.e., the difference between
TABLE II

PARAMETERS OF THE RBD AND CBD DETECTION METHODS.

\begin{tabular}{ccc}
\hline Method & Parameter & Description \\
\hline \multirow{2}{*}{ RBD } & $q$ & Length of the regressor \\
& $\tau$ & Time window of the prediction error \\
& $\xi$ & Threshold of the prediction error \\
\hline CBD & $\lambda$ & Time window for feature construction \\
\hline
\end{tabular}

$\tilde{g}_{j}$ and $g_{j}$ is as small as possible. To this end, as in the case of the RBD, a suitable training phase is performed offline to find the optimal values of the parameter vector $\underline{\alpha}$. Once the best model within the family $\Gamma$ has been found, at each time $t$ the detection whether a covert channel is present is performed online by feeding the trained model with the vector $\underline{f}_{t}$ of the current features and analyzing the value of the output $\tilde{g}_{t}$.

Differently from the RBD method, where the same approximate model is used to detect covert communications with all the seven techniques introduced in Section III, the CBD approach requires the training of a different model for each covert channel.

We point out that the accuracy of the CBD depends on the quality of the approximating model and on the parameter $\lambda$ used to construct the vector of features. Section VI will discuss its impact on the quality of detection in a real scenario. Notice that the CBD requires the tuning of only this parameter, whereas the RBD relies upon three parameters. Table II summarizes the main parameters of the two considered detection methods and their meaning.

\section{NUMERICAL RESULTS}

To evaluate the effectiveness of the proposed approach, we conducted experimental trials using two different smartphones, i.e., a Samsung Galaxy SIII and a LG Optimus 4X HD P880. We tested the RBD and CBD methods using Matlab with the Neural Networks and Statistics Toolboxes. All the experiments were done on a computer with a $2.5 \mathrm{GHz}$ Intel i7 CPU and 4 GB of RAM.

A dataset containing the consumption of all the software components running on the smartphones was generated using the methodology presented in Section IV. In more details, the consumption was measured with a per-process granularity, both in a "clean" set-up and when a local covert channel among two processes is created. Preliminary investigations showcased that the overall power consumption of the smartphone is well represented by the energy required by processes with OS-wide dynamics. More specifically, the energy consumption of the process System gave us enough condensed information to capture the general trend. Indeed, the System process is the core of all mechanisms required to access the low-level drivers of Android. It is used for manipulating notifications, display, audio, alarms, and telephony, just for naming a few. Therefore, for testing our methods, we relied upon the information provided solely by the process System.

As said, we focused on a scenario in which informationhiding-capable malware increases its stealthiness by acting when the device is idle, i.e., there is no user activity involved. 


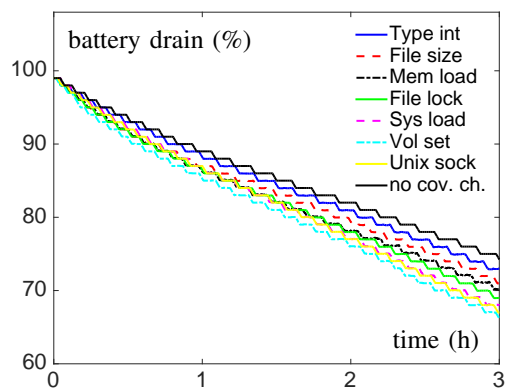

Fig. 4. Battery drain for each type of covert channel during 3 hours of repeated experiments.

As regards the exchanged messages, we set a fixed size of 1000 bytes, which is large enough to represent the exfiltration of sensitive information such as a bank account or a collection of contacts stored in the address book. Each covert channel was tested by performing 100 data transmissions starting at random time instants. To have a proper statistical relevance, each trial was repeated 10 times.

Figure 4 shows the trend of the battery drain for a period of 3 hours in the presence of colluding applications transmitting data with the seven implemented covert channels. Results indicate that the most consuming methods are the Volume Settings, the Memory Load, and the System Load, whereas the lowest one is the Type of Intent. Even if it is one of the most consuming, the System Load covert channel is also the one with the smallest bitrate.

The performances of the proposed detection methods were quantified by means of the percentage of correct detection of hidden communications, defined as follows:

$$
d=\frac{1}{T} \sum_{t=0}^{T-1} 1-\left|y_{t}-\tilde{y}_{t}\right|
$$

where $T$ is the length of each trial (dependent on the type of used covert channel) and $y_{t}$ and $\tilde{y}_{t}$ denote the actual and spotted hidden communications at time $t$, respectively. In more details, $y_{t}=1$ indicates that two colluding applications are covertly exchanging data, whereas $y_{t}=0$ represents the absence of hidden communication.

\section{A. RBD Method}

A training set composed of 5000 energy consumption measurements was used to optimize the parameters of neural networks and decision trees for the RBD method. Such samples were obtained in a clean system without hidden communication between colluding applications. The training was performed using the Levenberg-Marquardt algorithm [47] for neural networks and by minimizing the MSE of the predictions compared to the training data for decision trees, using the Gini's diversity index as split criterion [50]. As pointed out in Section V-B, the same approximator was used to detect all the seven implemented covert channels.

In order to determine the best values of the parameters $q$, $\xi, \tau$, and $\nu$ a thorough simulation campaign was performed. Concerning neural networks, Figure 5(a) reports the percentage of correct detection $d$ of each covert channel defined as

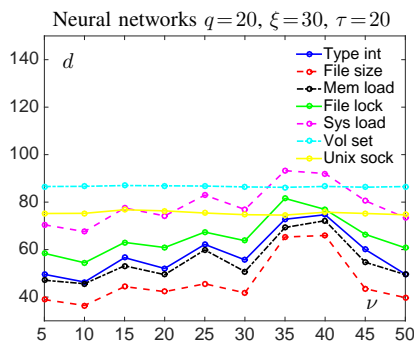

(a)

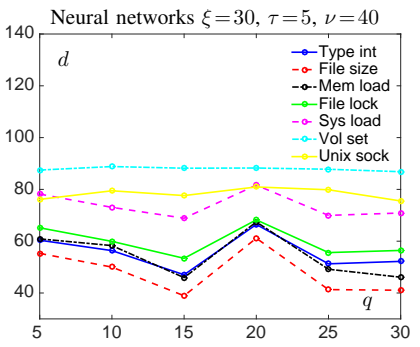

(b)

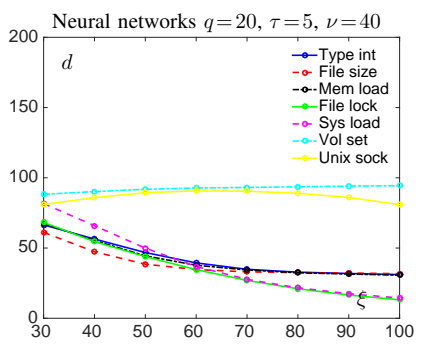

(d)

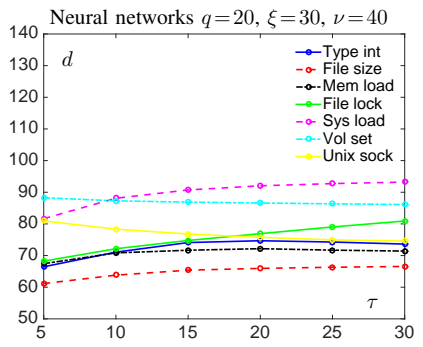

(f)

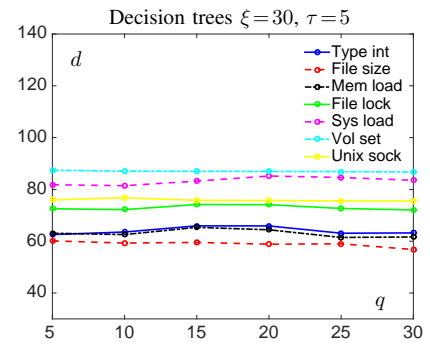

(c)

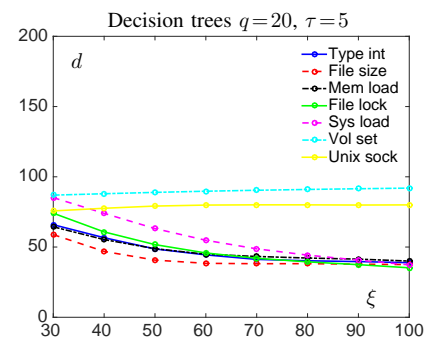

(e)

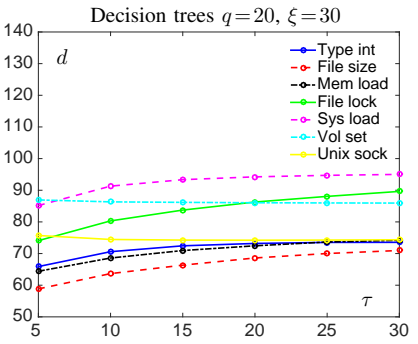

(g)
Fig. 5. Average percentage of correct detection for each covert channel using the RBD when varying the parameters of neural networks ( $a, b, d$, and $f$ ) and decision trees (c, e, and g).

in equation (4), averaged over 10 different trials, when $\nu$ is varied from 5 to 50 and the other parameters $q, \xi$, and $\tau$ are fixed to 20,30, and 20, respectively. Figures 5(b) and 5 (c) show the behavior of the average $d$ obtained with neural networks and decision trees, respectively, when varying the length $q$ of the regressor from 5 to 30 and with the other parameters fixed. Figures 5(d) and 5(e) depict the average $d$ using neural networks and decision trees, respectively, as a function of the threshold $\xi$ used for the detection rule, whereas the other parameters are fixed. Lastly, Figures 5(f) and 5(g) showcase the average $d$ obtained with neural networks and decision trees, respectively, as a function of the length $\tau$ of the time window used for the detection rule, with the other parameters fixed. 


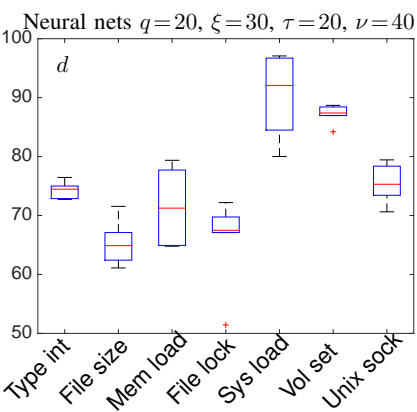

(a)

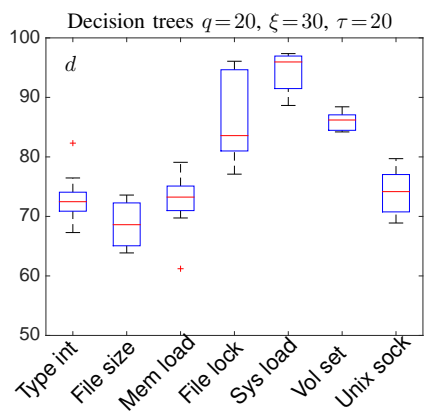

(b)
Fig. 6. Boxplots of the percentages of correct detection for each covert channel using the RBD with neural networks (a) and decision trees (b).

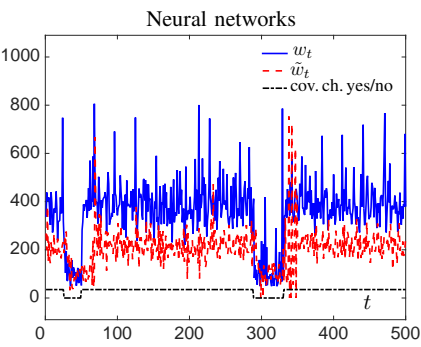

(a)

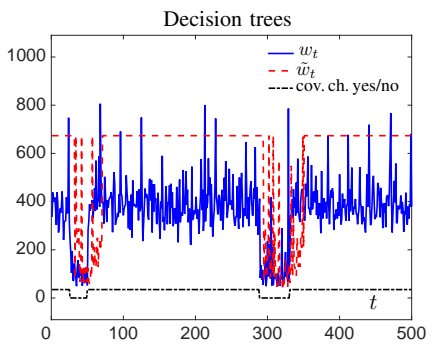

(b)
Fig. 7. Comparison of real and estimated power consumption of the System process for the Volume Settings by using the RBD with neural networks (a) and decision trees (b).

From the obtained results, it turns out that the percentage of correct detection for neural networks increases with $\nu$ up to $\nu=40$, which is then the best number of activation functions. For larger values, the phenomenon of overfitting is experienced, i.e., the number of basis functions is too large for the available data, and minor fluctuations in the energy measures may be overemphasized, thus resulting into bad detection rates. Concerning the length $q$ of the regressor, the best value turns out to be $q=20$. The behavior of neural networks is more affected by the chosen value if compared to decision trees, for which all the values of $q$ guarantee almost the same results. Instead, neural networks and decision trees exhibit the same behavior when varying the threshold $\xi$ for the prediction error (2), for which the best value appears to be $\xi=30$. Lastly, the percentage of correct detection grows if the time horizon $\tau$ used in (2) increases up to $\tau=20$, and then remains almost constant. Thus, in the perspective of saving computational time, $\tau=20$ is the best value.

Figure 6 shows the boxplots of the percentages of correct detection for each information-hiding technique computed over 10 different trials by using neural networks and decision trees with the best values of their parameters, i.e., $\nu=40$, $q=20, \xi=30$, and $\tau=20$. We conclude that the performance of neural networks and decision trees are comparable, i.e., on the average the accuracy of the detection is similar in both cases. The most easily detectable method appears to be the System Load covert channel, whereas the method that is least detectable is the File Size.

Figure 7 depicts the measured trend of the consumption of the System process compared with its estimation provided

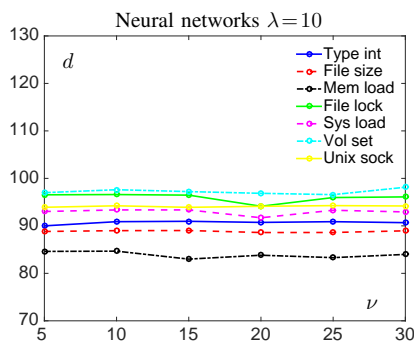

(a)

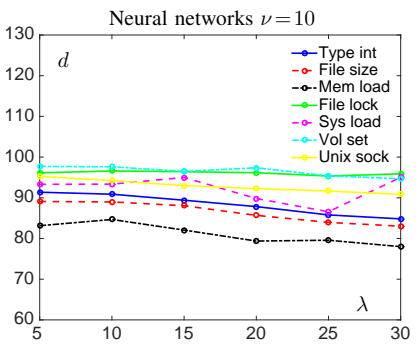

(b)

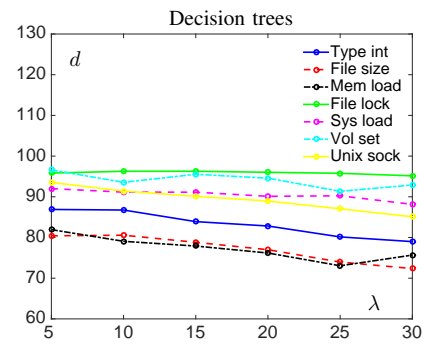

(c)
Fig. 8. Average percentage of correct detection for each covert channel using the CBD when varying the parameters of neural networks ( $a$ and $b$ ) and decision trees (c).

by neural networks and decision trees when using the Volume Settings covert channel. The presence or absence of hidden communication is denoted by high or low values of the binary signal at the bottom of each figure. As it can be seen, the prediction of the energy consumption is more accurate when no covert communication is active, whereas the prediction is not accurate in the presence of colluding applications. The "bad" prediction when covert channels are present is fundamental to spot hidden communications. More specifically, neural networks underestimate the power consumption, whereas decision trees saturate to a certain value. This is not surprising since the models have been built using a "clean" system without colluding applications.

\section{B. CBD Method}

To test the effectiveness of the CBD method, we used again a training set made up of 5000 energy samples. Differently from the RBD, the training was performed both when the colluding applications are active and inactive. Moreover, different approximators were trained for each of the seven implemented covert channels.

Since we had to solve a classification problem, the realvalued output of the neural networks was rounded either to 1 or 0 depending on whether hidden communication is spotted or not. Concerning decision trees, we adopted the so-called classification trees, whose output is directly one of the classes defined during the training. The training of neural networks was performed again by using the LevenbergMarquardt algorithm, whereas classification trees were trained by minimizing the MSE of the predictions compared with the trained data and using the Gini's diversity index as the split criterion.

For the case of neural networks, we varied both the number of neurons $\nu$ and the number of time instants $\lambda$ for the 


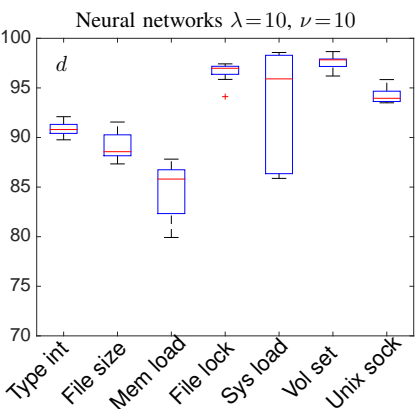

(a)

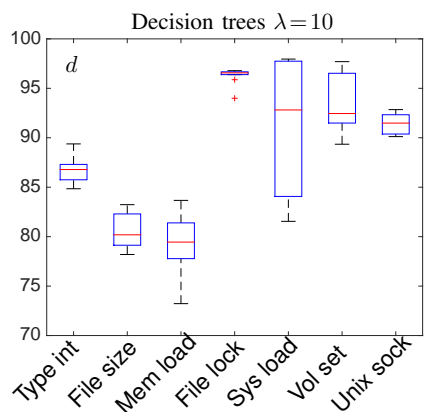

(b)
Fig. 9. Boxplots of the percentages of correct detection for each covert channel using the CBD with neural networks (a) and decision trees (b).

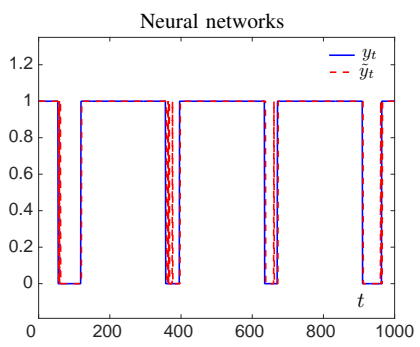

(a)

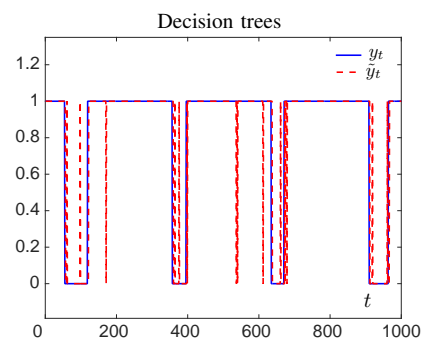

(b)
Fig. 10. Comparison of the true covert channel activity over time against the estimated one for the Volume Settings by using the CBD with neural networks (a) and decision trees (b).

computation of the features as in (3), in order to investigate their influence on the accuracy of the detection. Figure 8(a) depicts the percentage of correct detection $d$ of each covert channel, averaged over 10 different trials, when $\nu$ is varied from 5 to 30 and $\lambda$ is fixed to 10 . Figure $8(\mathrm{~b})$ presents the average $d$ for each information-hiding method when $\lambda$ ranges from 5 to 30 and $\nu$ equals to 10 . It turns out that the number of neurons affects the accuracy of the detection only marginally. Therefore, to save memory and computational time, one might choose $\nu=5$ or $\nu=10$. As regards the effect of $\lambda, \lambda=5$ or $\lambda=10$ appear to be the best choice since a small decay of performance is experienced for large values. Concerning decision trees, we investigated the effect of the parameter $\lambda$ on the accuracy of detection. The results are reported in Figure 8 (c) for the average $d$. Also in this case, $\lambda$ varies from 5 to 30 , and the percentage of detection decreases if $\lambda$ increases. Hence, optimal values are again $\lambda=5$ or $\lambda=10$.

Figure 9 depicts the boxplots of the percentages of correct detection for each covert channel computed over 10 different trials using the approximate models with the best values of their parameters, i.e., $\nu=10$ and $\lambda=10$ for neural networks and $\lambda=10$ for decision trees. In general, neural networks guarantee better performance compared with decision trees, i.e., on the average the accuracy of the detection is higher and with a lower variance. In all cases, the most easily detectable covert channels are the File Lock and the Volume Settings. Instead, the Memory Load method is the most difficult to detect despite its high consumption. Instead, the System Load is characterized by the largest dispersion.

Figure 10 portraits the estimated covert channel activity
TABLE III

AVERAGE PERCENTAGES OF CORRECT DETECTION FOR THE DIFFERENT DETECTION METHODS AND COVERT CHANNELS.

\begin{tabular}{ccccc}
\hline Covert channel & \multicolumn{2}{c}{ RBD } & \multicolumn{2}{c}{ CBD } \\
& Neural net. & Dec. tree & Neural net. & Dec. tree \\
\hline Type of Intent & 74.3 & 73.1 & 90.8 & 86.7 \\
File Size & 65.3 & 68.6 & 88.9 & 80.5 \\
Memory Load & 71.5 & 72.4 & 84.6 & 79.0 \\
File Lock & 65.9 & 86.2 & 96.5 & 96.2 \\
System Load & 90.4 & 94.2 & 93.3 & 91.1 \\
Volume Settings & 87.1 & 86.0 & 97.6 & 93.5 \\
Unix Socket & 75.4 & 74.2 & 94.2 & 91.4 \\
\hline
\end{tabular}

compared to the real one for the Volume Settings method. As it can be seen, neural networks and decision trees are able to correctly spot the channel activity most of the time, thus showcasing their effectiveness for run-time or static analysis purposes within the security framework of the device.

\section{Comparison Between RBD and CBD}

To sum up, Table III reports the percentage of correct detection averaged over 10 trials for all the implemented covert channels for the RBD and CBD methods. In both cases, the System Load and the Volume Settings are the most easily detectable covert channels. This may be ascribed to the fact that such methods are also the most power-consuming, i.e., their energy footprint is more evident. In this case, the hidden communication is correctly spotted 9 times over 10 on the average. The most difficult methods to detect turns out to be the File Size and the Memory Load. However, even if lower than the one of the best-performing methods, their average percentage of correct detection is about $65 \%$ when using the $\mathrm{RBD}$ and $85 \%$ for the $\mathrm{CBD}$, which is quite a satisfactory result. The Memory Load covert channel seems the most difficult to detect. This behavior is due to the absence of code in the System process that allocates memory: this task is done at high level by the Dalvik virtual machine and at low level by the Linux kernel.

According to the obtained results, in general the CBD outperforms the RBD in terms of percentage of correct detection. Moreover, it is worth noting that the RBD has three parameters to be tuned, i.e., $q, \tau$, and $\xi$, instead of only one for the CBD, i.e., $\lambda$. As a consequence, the implementation of the CBD in a production quality tool should be preferred, both in terms of complexity and performance.

Concerning the computational effort, the average time for the training was equal to 82.5 seconds for the RBD with $\nu=40$ and $q=20$ and to 15.6 seconds for the CBD with $\nu=10$ and $\lambda=10$ when using neural networks. The training times of the two detection methods when using decision trees with $q=20$ and $\lambda=10$ were equal to 1.92 and 0.27 seconds, respectively. The higher times of the RBD are mainly due to the greater dimension of the input vector compared to the CBD. In fact, in the first case the dimension of the input is equal to the length $q=20$ of the regressor, whereas in the second one it is equal to the number of features, i.e., 3. This also requires a larger number of neurons to obtain satisfactory approximations. In general, neural networks appears to be 
more computationally demanding compared to decision trees. Nevertheless, the RBD requires the training of only one model, whereas an approximate model for each covert channel is required by the $\mathrm{CBD}$, thus resorting to seven different training procedures.

The average time to spot the presence of a covert channel for the RBD method with the best values of the parameters is equal to 0.01 and 0.001 seconds, depending on whether neural networks or decision trees are used, respectively. As regards the CBD approach, such times when using the best values of the parameters are again equal to 0.01 and 0.001 seconds for neural networks and decision trees, respectively. In all cases, the online computational effort is very small. Thus, the proposed methods appear to be well-suited to being implemented in an online detection framework directly running on a mobile device.

\section{CONCLUSIONS AND Future WORKS}

In this paper we have presented a framework based on artificial intelligence tools, such as neural networks and decision trees, to detect the presence of malware using informationhiding techniques based on power measurements. Specifically, we have focused on the colluding application scenario, which is characterized by two processes trying to communicate outside their sandboxes for malicious purposes, for instance, for sensitive data exfiltration. Two detection methods have been developed, requiring the solution of regression and classification problems. To verify the effectiveness of our approach, we have implemented seven local covert channels on the Android platform, and we have performed an experimental measurement and detection campaign. The obtained results indicate that both methods are characterized by a good detection performance and can be used as an accurate IDS software on a modern smartphone to reveal the presence of hazards exploiting information hiding.

Future works aim at making our detection framework more effective, for instance by developing proper metrics to recognize at runtime the pair of colluding applications. Moreover, part of our ongoing research is devoted to understand if using additional information (e.g., activity correlation) could increase the accuracy of the approach. At the same time, we also work to extend the energy-based detection approach to other threats exploiting information hiding. On the overall, such improvements should lead to the development of an application directly running on a mobile device to spot the presence of covert communications in real-time.

\section{REFERENCES}

[1] K. Allix, Q. Jerome, T. F. Bissyande, J. Klein, R. State, and Y. le Traon, "A forensic analysis of Android malware-How is malware written and how it could be detected?" in Computer Software and Applications Conf., 2014, pp. 384-393.

[2] V. Rastogi, Y. Chen, and X. Jiang, "Catch me if you can: Evaluating Android anti-malware against transformation attacks," IEEE Trans. on Information Forensics and Security, vol. 9, no. 1, pp. 99-108, Jan. 2014.

[3] J.-F. Lalande and S. Wendzel, "Hiding privacy leaks in Android applications using low-attention raising covert channels," in Int. Conf. on Availability, Reliability and Security, 2013, pp. 701-710.

[4] W. Mazurczyk and L. Caviglione, "Steganography in modern smartphones and mitigation techniques," IEEE Communications Surveys \& Tutorials, vol. 17, no. 1, pp. 334-357, 2014.
[5] W. Mazurczyk and L. Caviglione, "Information hiding as a challenge for malware detection," Security \& Privacy, vol. 13, no. 2, pp. 89-93, 2015.

[6] McAfeeLabs, "McAfee labs threat report," August 2014. [Online]. Available: http://www.mcafee.com/uk/resources/reports/rp-quarterly-threat-q2-2014.p

[7] J. Hoffmann, S. Neumann, and T. Holz, "Mobile malware detection based on energy fingerprints - a dead end?" in Research in Attacks, Intrusions, and Defenses. Springer, 2013, pp. 348-368.

[8] L. Zhang, B. Tiwana, Z. Qian, Z. Wang, R. P. Dick, Z. M. Mao, and L. Yang, "Accurate online power estimation and automatic battery behavior based power model generation for smartphones," in Proc. Int. Conf. on Hardware/Software Codesign and System Synthesis, 2010, pp. $105-114$.

[9] L. Caviglione and A. Merlo, "The energy impact of security mechanisms in modern mobile devices," Network Security, vol. 2012, no. 2, pp. 1114, 2012.

[10] A. Merlo, M. Migliardi, and P. Fontanelli, "On energy-based profiling of malware in Android," in Int. Conf. on High Perf. Computing \& Simulation, 2014, pp. 535-542.

[11] C. Marforio, H. Ritzdorf, A. Francillon, and S. Capkun, "Analysis of the communication between colluding applications on modern smartphones," in Proc. Annual Computer Security Applications Conf., 2012, pp. $51-60$.

[12] A. Merlo, M. Migliardi, and P. Fontanelli, "Measuring and estimating power consumption in Android to support energy-based intrusion detection," Journal of Computer Security, vol. 23, pp. 611-637, 2015.

[13] S. Haykin, Neural Networks, A comprehensive foundation. Prentice Hall, 1999.

[14] L. Breiman, J. Friedman, R. Olshen, and C. Stone, Classification and Regression Trees. Chapman \& Hall, 1984.

[15] A. Bose, X. Hu, K. G. Shin, and T. Park, "Behavioral detection of malware on mobile handsets," in Proc. Int. Conf. on Mobile Systems, Applications, and Services, 2008, pp. 225-238.

[16] A. Shabtai, R. Moskovitch, Y. Elovici, and C. Glezer, "Detection of malicious code by applying machine learning classifiers on static features: A state-of-the-art survey," Information Security Technical Report, vol. 14, no. 1, pp. 16-29, 2009.

[17] A. Shabtai, U. Kanonov, Y. Elovici, C. Glezer, and Y. Weiss, "Andromaly: a behavioral malware detection framework for Android devices," Journal of Intelligent Information Systems, vol. 38, no. 1, pp. 161-190, 2012.

[18] L. Liu, G. Yan, X. Zhang, and S. Chen, "VirusMeter: Preventing your cellphone from spies," in Recent Advances in Intrusion Detection, 2009, pp. 244-264.

[19] G. Jacoby and N. Davis, "Battery-based intrusion detection," in IEEE Global Telecommunications Conf., vol. 4, 2004, pp. 2250-2255.

[20] D. C. Nash, T. L. Martin, D. S. Ha, and M. S. Hsiao, "Towards an intrusion detection system for battery exhaustion attacks on mobile computing devices," in IEEE Int. Conf. on Pervasive Computing and Communications Workshops, 2005, pp. 141-145.

[21] H. Kim, J. Smith, and K. G. Shin, "Detecting energy-greedy anomalies and mobile malware variants," in Proc. Int. Conf. on Mobile Systems, Applications, and Services, 2008, pp. 239-252.

[22] B. Dixon, Y. Jiang, A. Jaiantilal, and S. Mishra, "Location based power analysis to detect malicious code in smartphones," in Proc. Workshop on Security and Privacy in Smartphones and Mobile Devices, 2011, pp. 27-32.

[23] B. Dixon and S. Mishra, "Power based malicious code detection techniques for smartphones," in IEEE Int. Conf. on Trust, Security and Privacy in Computing and Communications, 2013, pp. 142-149.

[24] B. Dixon, S. Mishra, and J. Pepin, "Time and location power based malicious code detection techniques for smartphones," in IEEE Int. Symp. on Network Computing and Applications, 2014, pp. 261-268.

[25] M. Curti, A. Merlo, M. Migliardi, and S. Schiappacasse, "Towards energy-aware intrusion detection systems on mobile devices," in Int. Conf. on High Perf. Computing and Simulation, 2013, pp. 289-296.

[26] T. K. Buennemeyer, G. Jacoby, W. G. Chiang, R. C. Marchany, and J. G. Tront, "Battery-sensing intrusion protection system," in Information Assurance Workshop, 2006, pp. 176-183.

[27] T. K. Buennemeyer, T. M. Nelson, M. Gora, R. C. Marchany, and J. G. Tront, "Battery polling and trace determination for bluetooth attack detection in mobile devices," in Information Assurance and Security Workshop, 2007, pp. 135-142.

[28] L. Caviglione, A. Merlo, and M. Migliardi, "What is green security?" in Int. Conf. on Information Assurance and Security, 2011, pp. 366-371. 
[29] F.-E. Kioupakis and E. Serrelis, "Preparing for malware that uses covert communication channels: The case of Tor-based Android malware," in Int. Conf. Information Security and Digital Forensics, 2014, pp. 85-96.

[30] P. Faruki, V. Ganmoor, V. Laxmi, M. S. Gaur, and A. Bharmal, "AndroSimilar: robust statistical feature signature for Android malware detection," in Proc. Int. Conf. on Security of Information and Networks, 2013, pp. 152-159.

[31] R. Andriatsimandefitra and V. V. T. Tong, "Detection and identification of Android malware based on information flow monitoring," in Int. Conf. on Cyber Security and Cloud Computing, 2015, pp. 1-4.

[32] R. Schlegel, K. Zhang, X. Zhou, M. Intwala, A. Kapadia, and X. Wang, "Soundcomber: A stealthy and context-aware sound trojan for smartphones," in NDSS, vol. 11, 2011, pp. 17-33.

[33] A. Merlo, M. Migliardi, and L. Caviglione, "A survey on energy-aware security mechanisms," Pervasive and Mobile Computing, vol. 24, pp. 77-90, 2015.

[34] A. Armando, A. Merlo, M. Migliardi, and L. Verderame, "Breaking and fixing the Android launching flow," Computers and Security, vol. 39, pp. 104-115, 2013.

[35] A. Armando, A. Merlo, and L. Verderame, "An empirical evaluation of the Android security framework," in Security and Privacy Protection in Information Processing Systems, ser. IFIP Advances in Information and Communication Technology, L. Janczewski, H. Wolfe, and S. Shenoi, Eds. Springer Berlin Heidelberg, 2013, vol. 405, pp. 176-189.

[36] S. Lee, W. Jung, Y. Chon, and H. Cha, "EnTrack: a system facility for analyzing energy consumption of Android system services," in Proc. Int. Joint Conf. on Pervasive and Ubiquitous Computing, 2015, pp. 191-202.

[37] T. Hastie, R. Tibshirani, and J. Friedman, The Elements of Statistical Learning (2nd Ed.). New York: Springer, 2009.

[38] R. Zoppoli, T. Parisini, and M. Sanguineti, "Approximating networks and extended Ritz method for the solution of functional optimization problems," J. of Optimization Theory and Applications, vol. 112, pp. 403-439, 2002.

[39] M. Gaggero, G. Gnecco, and M. Sanguineti, "Dynamic programming and value-function approximation in sequential decision problems: error analysis and numerical results," Journal of Optimization Theory and Applications, vol. 156, no. 2, pp. 380-416, 2013.

[40] M. Gaggero, G. Gnecco, and M. Sanguineti, "Approximate dynamic programming for stochastic N-stage optimization with application to optimal consumption under uncertainty," Computational Optimization and Applications, vol. 58, no. 1, pp. 31-85, 2014.

[41] L. Caviglione, "Enabling cooperation of consumer devices through peerto-peer overlays," IEEE Trans. Consumer Electronics, vol. 55, no. 2, pp. 414-421, 2009.

[42] A. Alessandri, C. Cervellera, and M. Gaggero, "Nonlinear predictive control of container flows in maritime intermodal terminals," IEEE Trans. Contr. Syst. Technol., vol. 21, no. 4, pp. 1423-1431, 2013.

[43] K. Hornik, M. Stinchombe, and H. White, "Multilayer feedforward networks are universal approximators," Neural Networks, vol. 2, pp. 359-366, 1989.

[44] A. Barron, "Universal approximation bounds for superpositions of a sigmoidal function," IEEE Trans. Inf. Theory, vol. 39, no. 3, pp. 930 945, 1993.

[45] R. A. Berk, Statistical Learning from a Regression Perspective. New York: Springer-Verlag, 2008.

[46] C. Cervellera, M. Gaggero, and D. Macciò, "An analysis based on Fdiscrepancy for sampling in regression tree learning," in Proc. Int. Joint Conf. on Neural Networks, 2014, pp. 1115-1121.

[47] D. W. Marquardt, "An algorithm for least-squares estimation of nonlinear parameters," Journal of the Society for Industrial \& Applied Mathematics, vol. 11, no. 2, pp. 431-441, 1963.

[48] P. Werbos, The Roots of Backpropagation: From Ordered Derivatives to Neural Networks and Political Forecasting. New York, NY, USA: Wiley-Interscience, 1994.

[49] A. Sen and M. Srivastava, Regression Analysis - Theory, Methods, and Applications. Springer-Verlag, 2011.

[50] T. Cover and T. Joy, Elements of Information Theory. Wiley, 1991.

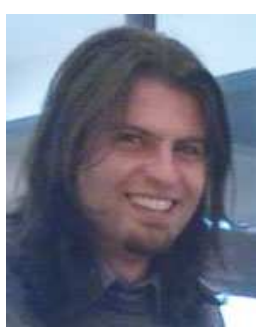

Luca Caviglione received the Ph.D. degree in electronics and computer engineering from the University of Genoa, Genoa, Italy. He has been involved in research projects funded by ESA, EU, and MIUR. He is currently a Research Scientist with the Institute of Intelligent Systems for Automation, National Research Council of Italy, Genoa. He is a Work Group Leader of the Italian IPv6 Task Force, a Contract Professor, and a Professional Engineer. He has authored or co-authored over 90 academic publications, and several patents. His current research interests include P2P systems, wireless communications, cloud architectures, and network security. Dr. Caviglione is involved in the technical program committee of many international conferences and regularly serves as a Reviewer for the major international journals. Since 2011, he has been an Associate Editor of Transactions on Emerging Telecommunications Technologies (Wiley).

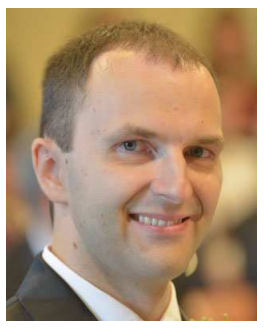

Mauro Gaggero received the B.Sc. and M.Sc. degrees in electronics engineering and the Ph.D. degree in mathematical engineering from the University of Genoa, Genoa, Italy, in 2003, 2005, and 2010, respectively. He was a Post-Doctoral Fellow with the Faculty of Engineering, University of Genoa, in 2010. Since 2011, he has been a Research Scientist with the Institute of Intelligent Systems for Automation, National Research Council of Italy, Genoa. His current research interests include control and optimization of nonlinear systems, distributed parameter systems, neural networks, and learning from data. Dr. Gaggero is an Associate Editor of the European Control Association Conference Editorial Board and of the IEEE Control Systems Society Conference Editorial Board.

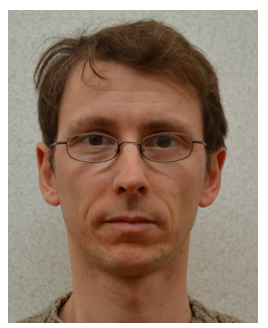

Jean-François Lalande received the Ph.D. degree in computer science from Inria, SophiaAntipolis, France, within the Mascotte Project (CNRS/Inria/UNSA) in 2004. He is currently an Associate Professor with the INSA Centre Val de Loire, in the Laboratoire d'Informatique de l'Université d'Orléans (LIFO). He is also temporarily associated with Inria in the CIDRE team. During the Ph.D. his research interests focused on the combinatorial optimization for optical and satellite networks. Since 2005 , he has been working on security of operating systems, C-embedded software (including smart cards), and Android applications. Currently, he is interested in mobile software security. Prof. Lalande actively participates to the release of open-source software in order to make security experiments reproducible.

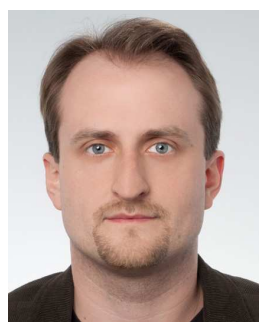

Wojciech Mazurczyk (SM'13) received the B.Sc., M.Sc., Ph.D. (with honors), and D.Sc. (habilitation) degrees in telecommunications from the Warsaw University of Technology (WUT), Warsaw, Poland, in 2003, 2004, 2009, and 2014, respectively. He is currently an Associate Professor with the Institute of Telecommunications at WUT, where he is the Head of the Bio-inspired Security Research Group (bsrg.tele.pw.edu.pl). His research interests include bio-inspired cybersecurity and networking, information hiding, and network security. Prof. Mazurczyk is involved in the technical program commitee of many international conferences, including IEEE INFOCOM, IEEE GLOBECOM, IEEE ICC, and ACSAC. Also, he serves as a Reviewer for the major international magazines and journals. Since 2013, he is an Associate Technical Editor of the IEEE Communications Magazine (IEEE Comsoc).

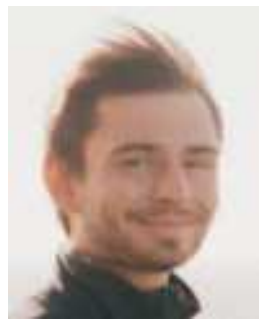

Marcin Urbański received the B.Eng. in computer science from the Warsaw University of Technology (WUT), Warsaw, Poland, in 2015. He currently works at the Norwegian University of Science and Technology, Trondheim, Norway, where he is implementing applications for interactive lectures. His research interests include steganography and mobile software security. 